\title{
Intelligent Agent based System for Crop Monitoring
}

\author{
Ahad Hanif ${ }^{1}$, Aslam Muhammad ${ }^{2}$, Ana María Martinez-Enriquez ${ }^{3}$, \\ Adrees Muhammad ${ }^{4}$ \\ ${ }^{1}$ Department of Mechatronics Engineering, UET, Lahore, Pakistan \\ ahad.hanif@umt.edu.pk \\ 2 Department of CS, UET, Lahore, Pakistan \\ maslameuet.edu.pk \\ ${ }^{3}$ Department of CS, CINVESTAV, CDMX, Mexico \\ ammartinecinvestav.mx \\ ${ }^{4}$ Department of CS, Superior University, Lahore, Pakistan \\ adreesgujer@gmail.com
}

\begin{abstract}
This paper describes the utility of environmental sensors for the monitoring of crops remotely, grown in a green house or a tunnel. The work combines the instrumentation techniques with knowledge based system. Agriculturalists frequently visit their farms to monitor the condition of plants and crop. This monitoring is time consuming. Conventional farms monitoring techniques and inspections give low crop production, attack of severe diseases parasites. Adequate amount of water, fertilizers, and nutrients can improve the yield and at the same time reduces the cost. In order to assist farmers and landowner, we designed and developed an agent based crop monitoring system. The objective of this research is to develop an agent based system to help the agriculturists to monitor the crops remotely by using recent technology and hence advise the farmers to take appropriate measures. The system is divided into main two main parts. First one is data acquisition and the other one is recommendation part. The readings of crucial environmental parameters are taken and sent to a central computer service. Color processing techniques are used to find the nitrogen deficiency in the green plants. Manual leaf color chart has been replaced by electronic one which is capable of providing sufficient information about future scenario of nitrogen content in the plants. The system uses real time information and recommends an appropriate solution of the problem caused by environmental stresses. In case of any critical situation, the system generates warnings in the form of an email, to be sent to the landowners or agriculturist.
\end{abstract}

Keywords: remote sensing, knowledge base, color processing, leaf color charts, intelligent agent.

\section{Introduction}

As the requirements of the researchers in the area of crop sciences change, a need is felt for developing the tools that assists them with their day to day activities. The system required for such a purpose needs to be intelligent in nature regarding the acquisition of data from the sensors, interfacing with the system and manipulating it 
in certain cases. Remote Sensing is the science and art of obtaining information about an object, area or phenomenon through the analysis of data acquired by a device that is not in contact with the object, area or phenomenon under investigation. Many systems of monitoring like RADARs, Satellite imaging and aerial photography exist but these systems are quite expensive, complex and require technical expertise to operate them. The environmental parameters are monitored and relayed to the monitoring system. The monitored parameters include temperature, humidity and light [1]. By maintaining optimum range of parameters like light, temperature and humidity one can protect the plants from pests and diseases [2]. Excessive water and low soil oxygen damages the roots of the plants [3].

Manual monitoring of crop depends on the knowledge of the farmers. The problem arises when farmer is not well educated and he needs the expert's opinion. Furthermore, manual monitoring is time consuming and ends up with low yield. It is not possible for the farmer to make sure his presence in the field round the clock. We have to monitor the crops/plants in real time. There are two main factors, which are crucial for the development of plants. They are crop water status and availability of fertilizers to meet the requirements of essential nutrients. Unsuitable ranges of environmental factors (temperature, humidity) disturb the mobility of nutrients in the plants. Plants may suffer from dehydration, chilling and freezing effects. They become vulnerable against the attack of pests and fungus.

Leaf color charts are being used by the farmers to check the nitrogen deficiency in the plants. Manual leaf color charts require personal technical expertise. If plants are supplied with insufficient amount of nutrients, then yield will be low. In other case production cost will be increased. Small scale farming requires a system which is not only cost effective but also provides good yield.

This paper proposes intelligent agent based system for monitoring the crops, which are located in the remote areas. It was designed for the acquisition of necessary parameters which are crucial for the development of the plants like humidity and temperature of the environment. The intelligent agent manipulate the data acquired by the sensors and also provide us with a suitable solution of the problem based on the expert's opinion. Green houses and tunnel farming techniques would be more productive if they are equipped with such a remote monitoring system. It gives suggestions to the farmers so that they take appropriate measures before the damage is done to the crop. In this work, all the knowledge regarding the normal growth of the plants is gathered within the knowledge base of the system. System utilizes this knowledge base while analysing the information and taking decisions.

The rest of the paper has four main parts. It includes the literature review, which provides insight into the existing woke done in Phytomonitoring techniques. Then comes the Materials and Methodology section, which elaborates our contribution in crop monitoring. Next section is about experimental results and discussions. The last section of the paper is conclusion, which summarizes the whole work.

\section{Literature Review}

Phytomonitoring techniques are very helpful when it comes to detect the physiological disorders in the plants. Environmental parameters including 
temperature, air humidity and soil moisture are recorded through sensors. For remote data transmission GSM modem is used [4] [5]. Growth rate of trunk, flower and fruits are most sensitive parameters in Phytomonitoring which may change with a slight modification in water balance. It is important to monitor and control the environmental parameters in real time to help the farmers to bring innovation in the irrigation strategy [6]. Image processing is now being used extensively in agriculture sector. Image processing may be used to find the age of wheat crop. The information was quite helpful in providing the suitable amount of fertilizers and nutrients to the plants. Overall cost reduces by the application of this technique [7]. In image processing, three prime colors combine to form an image. They are red, green and blue [9]. Rodríguez (2002) proposed hierarchical control of green house. Fungus damages the fruit. Its growth can be controlled by maintaining suitable ranges of temperature and humidity. So fruits storage properties were improved [8].

A trapezoidal two dimensional index was empirically derived by Thomas, which could detect water stress even with a low percentage of canopy cover [11]. Continuous monitoring and scheduling the irrigation process by using Infrared images have provided early warning of water stress [10]. The best way to find the plant water status is to detect the soil water content by evapotranspiration estimation. Infrared images were produced to find the vegetation index [12]. Solar energy based green house $(\mathrm{GH})$ monitoring system was designed for the monitoring of green house. All the nodes and sensors were powered by solar energy to solve the low battery issue. Data was transferred through ZigBee and GSM technology [13].

With the increase in world population we need more food to meet the requirements of the people. Many people die each year due to starvation. We need techno-economic solution of this problem. Conventional monitoring systems are not techno-economic. So much space is available to develop such a system which is cheaper, reliable and give quick response.

The objective of this research is to develop such a system which suggests the farmers and agriculturists, a solution of the problem caused by environmental stresses so that they take appropriate measures before the damage is done to the crop. The knowledge base of the system possesses the expertise of the experts. We would be able to maintain large amount of information which is quite helpful in making reasoning and rules. We have tried to bring flexibility in Leaf Color Chart (LCC) so that ever person can use it conveniently.

\section{Materials and Methodology}

The most crucial atmospheric element, which effects the growth and yield of plants is temperature. Optimum temperature is required for photosynthesis respiration. Second important parameter is humidity. Relative humidity is the ratio of two water vapour contents i.e. the real water vapour contents and saturated water vapour contents in the air. Both values are taken at same pressure \& temperature. It is expressed in percentage. Plants use air carbon dioxide for their photosynthesis process. Plant's leaves have pores to take in $\mathrm{CO} 2$. During respiration, some moisture goes out in the air through these pores. If air has high humidity, then plants will transpire moisture more slowly. The reverse happens when air has low moisture contents. Therefore, 
when air is dry then more water is evaporated from the plants. In this case plants become deficient in water and leaves close their pores. Although water outgoing is reduced but at the same time it reduces the Carbon dioxide intake. Plants absorb water through roots from soil. If evapotranspiration rate is high than absorption rate, then plants under go severe atmospheric and chemical stresses. It may cause the plant cells to die.

A sensors based crop monitoring system was developed which utilizes the field sensors to acquire information about the environmental parameters. It possesses the knowledge of the experts and provides farmers with a solution of the problem. Secondly, manual leaf color charts (LCC) have been replaced by electronic leaf color charts, which are not only flexible but are also easy to use. It assists the farmers to use the fertilizers in appropriate amount to minimize the production cost. Temperature sensor (DS18B20) and humidity sensor (HSU-04) were used to monitor the environment of the enclosed area in which plants were grown. IP camera was installed in the field to get the images of the plants to be compared with the standard leaf color chart.

The agent is basically a software which is designed to utilize the intelligence to carry out an assigned task automatically like retrieving and delivering information. Intelligent agent is also closely related to software agent (an autonomous computer program that carries out tasks on behalf of users). In computer science, the term intelligent agent may be used to refer to a software agent that has some intelligence. For example, an autonomous program, set for operator assistance or data mining, is also called an intelligent agent. An intelligent system incrementally accommodates new problem solving rules. It should have the capacity to analyze itself, behavior, faults and corrections. An agent maps every possible percept to an action. An agent perceives from its environment and surroundings through sensors and reciprocates through actuators.

The agent collects real time data and manipulates it by using its knowledge base and rules. Information about environmental parameters was transferred to a computer through RS-232 serial port. All the data is manipulated by the intelligent agent based system. The system monitors the temperature, humidity and the quantity of nutrients (Nitrogen) required for normal growth of the plants. Real time images of plants are taken by camera to find the nitrogen deficiency in the plants. The results are transferable through an email. There are two basic parts in this system. One is remote monitoring unit (RMU) and the other one is data manipulation unit (DMU). The color of the leaf changes with the change in the amount of nutrients. There are three main nutrients which are called prime nutrients. They are Nitrogen, phosphorous and potassium. Green color of leaf is due to nitrogen. If plant is deficient in nitrogen, then the color of the leaf becomes pale green. In addition, if plant has sufficient amount of nitrogen then leaves appear in dark green color. Real time images of plants are compared with a standard color scheme to find out the percentage of nitrogen in the plants.

Information from field sensors is transferred to the computer through a microcontroller (PIC18F452) and RS-232 serial port. Computer has an intelligent agent based system which utilizes this information. For better visualization, values which are coming from field sensors are also displayed on a 16x2 LCD. Agent, Matlab program, uses this information and on the bases of rules it generates an email 
which is sent to the user inbox to intimate him about the current situation. RS-232 Data Logger software has been incorporated. It generates a text file of the value of temperature and humidity. This file is automatically updated after regular intervals. This file is used by the Matlab code to apply the rules. System architecture is shown in Figure 1.

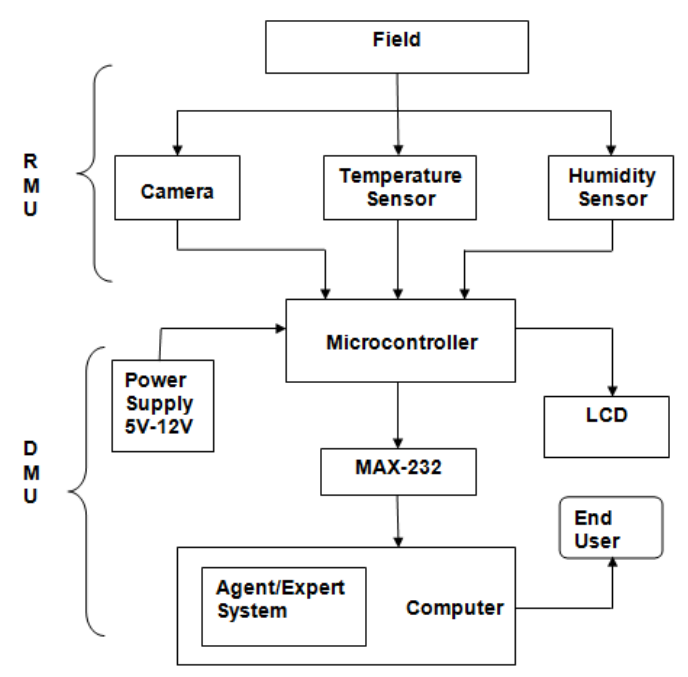

Fig. 1. System architecture.

A graphical user interface (GUI) has been developed to set the ranges for both temperature and humidity. Till the real time values remain between the ranges, no intimation or warning email is generated. But as soon as the value goes out of the range an email is generated, intimating the farmer about the unwanted conditions. Screen shot of GUI window is shown in Figure 2.

While doing color image processing we have to separate the desired color, which is under observation, from the original image. In our case we are working on the green color.

So firstly we have to identify the green color in the original image. There are many disturbing factors like sun shine, shades, background and presence of non-green colors in the original image which create hurdles in detecting the pure green color.

A technique has been developed to identify the green color in the image. Mask of the required color is created by using image processing tools and apply it to the original image. The program does the simple color detection in RGB color space. We first find out the individual color bands from the image. Histogram of the image is computed first and color threshold ranges are selected. This mask is now applied to the original image. After applying we can identify the green portion in the original image. The processed image is then compared with the standard leaf color chart.

Leaf color chart provides a very simple technique to check the nitrogen deficiency in the plants. It provides farmers with an opportunity to find out the plant's nitrogen demand so that they can apply the nitrogen fertilizer in appropriate amount to minimize the production cost. There are usually 6 shades of green colors on LCC 
which vary from light green to dark green. Each shade represents a specific amount of nitrogen deficiency. Different shades of green color are shown in Figure 3.

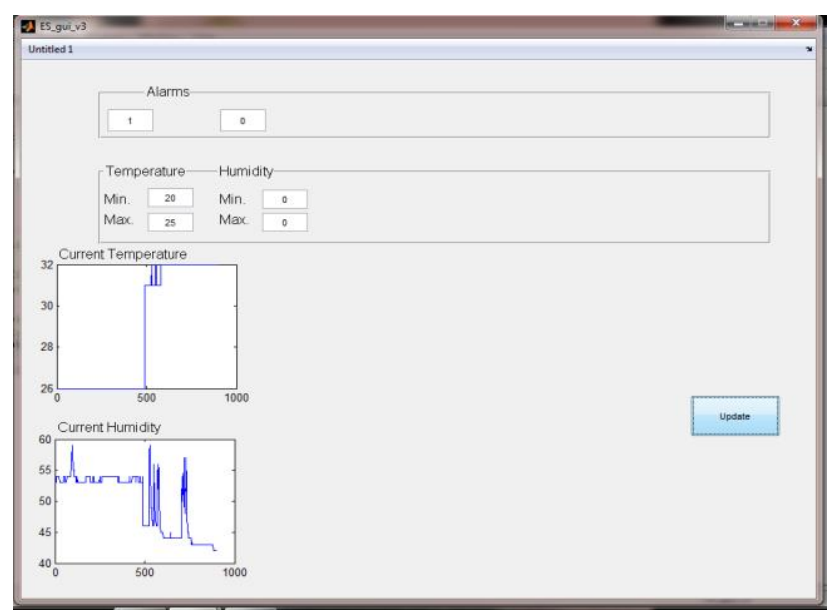

Fig. 2. GUI window.

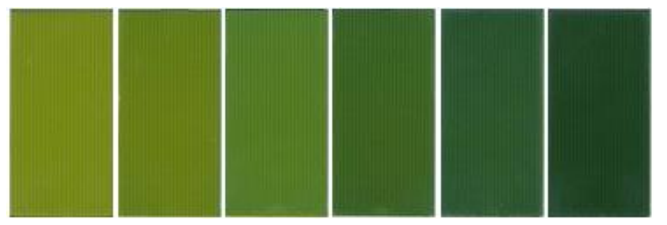

Fig. 3. Color scheme of LCC.

Light green color means that plants have high deficiency of nitrogen and dark green color means that plants have sufficient amount of nitrogen. Sometimes it happens that the color of leaf lies between the two shades and does not completely match with any shade. This issue has been addressed in this work. Electronic LCC gives actual comparison and tells if the color lies between the two shades.

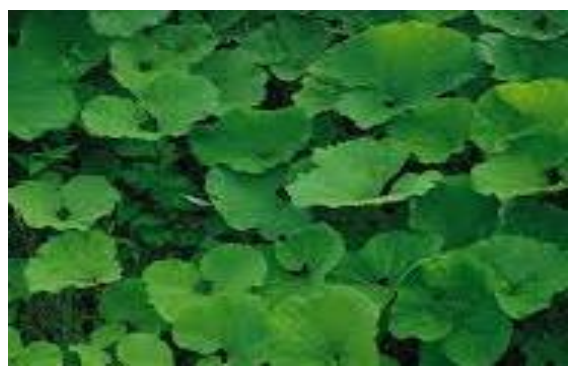

Fig. 4. Image from field.

Figure 4 shows the field image. It is compared with the standard color scheme. But before comparing we have to use some image processing tools to extract the green 
color from the image as described above. In this way, we can minimize the effects of non-green parts on the results.

\section{Experimental Results and Discussions}

There are total 256 pixels in the image. The range is from 0 to 255 . Each color has its own histogram. Histograms of all bands 'red, green and blue' are shown in Figure 5 (a) and 5 (b) respectively.

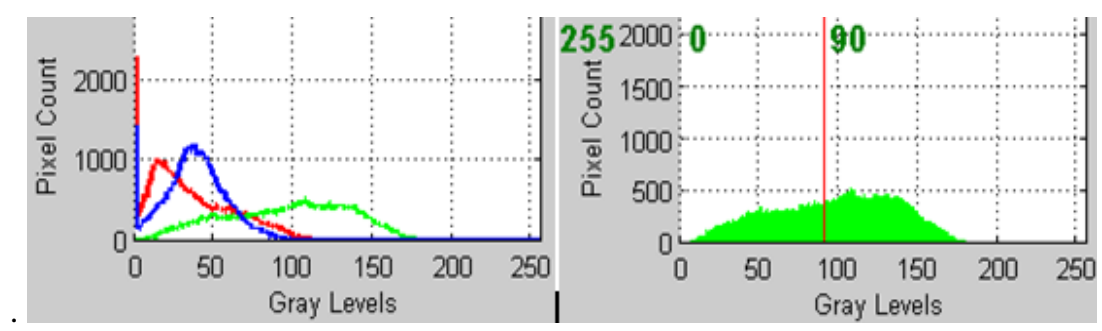

Fig. 5. (a) Histogram of all bands (b) Histogram of green band.

After the identification of green color we compared it with the standard ELCC. Shades of ELCC may vary depending upon the type of crop and the area in which it is grown. Therefore, for a specific region and crop, reference images for ELCC are collected first. These images make a color scheme. Now user can monitor the nitrogen contents in the plants by comparing the real time image with the standard color chart. Each pixel of field image is divided by the average value of reference images. But before finding the average value we have to use image processing tools as described above to find out the true green color from the image. The results are shown in Figure 6.

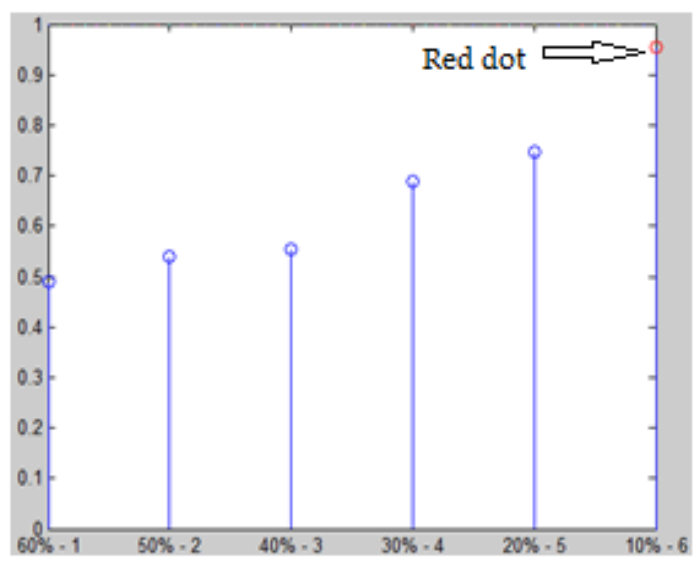

Fig. 6. Results of color processing. 
$\mathrm{X}$-axis is labeled as the percentage of nitrogen deficiency in the plants. There are six shades of green color in ELCC so there are 6 vertical lines, representing different shades of green color. The line, with red circle at top, is closest to the dotted horizontal line (passing through y-axis 1). This vertical line shows that the field image matches with that corresponding shade. If the circle is on the dotted line, it means that the corresponding shade from the scheme exactly matches the field image. If it is below or above the dotted line it means that the color of field image lies between the two shades. On $\mathrm{x}$-axis we can find that how much nitrogen deficiency is there in the plants.

On the basis of that information the agent suggests the amount of nitrogen fertilizer (like urea) to be added in the soil. Electronic leaf color chart (ELCC) provides us cost effective, efficient and quick measurement of nitrogen deficiency in the green plants. Intensity of Green color is associated with nitrogen contents. Sun light is a disturbing factor when camera takes the images of the plants. It may cause slight difference in color. We need to check whether camera is facing or not facing the sun light. If a picture is taken in sunlight, then it is possible that it shows different shade as compared to one taken in overcast weather.

Through this technique we have tried to minimize the effects of sun light and other factors. For better results, it is essential that camera should focus on green portion of the plants and should not include the non green parts like the color of trunk or soil etc.

\section{Conclusion}

In addition to remotely monitoring and controlling the plant environment, the system minimizes the human efforts and helps the farmers to produce techno-economical crops by utilizing nutrients in appropriate amount and minimizing the energy consumption.

The growth of plants becomes more sophisticated. By applying modern instrumentation and artificial Intelligence techniques in agriculture sector we can make this sector more productive and efficient. This system may also be used as testing tool by deliberately adjusting the water supply, temperature and humidity. We can analyze the impacts of environmental stresses on the growth of plants. In addition to that it is quite helpful to see the past trends of nitrogen deficiency is the plants. Results have been improved by the application of image processing tools.

To avoid any abrupt fluctuation in temperature and humidity value, agent takes the average of the previous values. So it avoids temporary alarming conditions. Real time monitoring of the plants through color image processing has made the system quite useful. Farmers are provided with the best possible solution through email.Plants can be grown in those areas which do not have suitable environmental conditions for a specific crop.

The results of this system could be used for future research especially by those departments which are concerned with data acquisition. It would be a great contribution if the system enhances its knowledge base automatically to compensate those conditions and rules which are not incorporate in its knowledge base. 


\section{References}

1. Zuo, X. et al.: Design of Environmental Parameters Monitoring System for WatermelonSeedlings Based on Wireless Sensor Networks. App Mathe. \& Infor. Sci., 243S250S (2011)

2. Albright L. D. et al.: Environmental Control for Plants on Earth and Space. IEEE Control Systems Magazine 21(5), 28-47 (2011)

3. Schaffer, B.: Effects of Soil Oxygen Deficiency on Avocado (persea americana mill.) Trees, Seminario International. En: Manejo del Riego y Suelo en el Cultivo del Palto La Cruz, Chile (2006)

4. Avidan, A., Hazan, A.: Application of the Phytomonitoring Technique for Table Grapes. Submitted for the Proceedings of the International Workshop on Advances in Grape vine and Wine Research. Venosa (Italy), September 15-17 (2005)

5. Puig, V. et al.: Optimal predictive control of water transport systems: Arrêt-Darré/Arros, a case study. Water Sci Technol 60(8), 2125-33 (2009)

6. Ton, Y., Kopyt, M.: Phytomonitoring in Realization of Irrigation Strategies for Win grapes. Acta Hort. (ISHS) 652, pp. 167-173 (2004)

7. Kakran, A., Mahajan, R.: Monitoring Growth of Wheat Crop using Digital Image Processing. International Journal of Computer Applications 50(10) (2012)

8. Ibrahim, M., Rabah, A.B.: Effect of Temperature and Relative Humidity on the Growth of Helminthosporium fulvum. Nigerian Journal of Basic and Applied Science 19(1), 127$129(2011)$

9. Plataniotis, K.N., Venetsanopoulos, A.N.: Color Image Processing and Applications. (2000)

10. Rodríguez, F.: Modeling and Hierarchical Control of Greenhouse Crop Production (in Spanish). PhD thesis, University of Almería, Spain (2000)

11. Kopyt, M., Ton, Y.: Phytomonitoring Technique for Table Grapes Application Guide. $2^{\mathrm{ND}}$ Edition, PhyTech Ltd. (2005)

12. Clarke, T. R.: An Empirical Approach for Detecting Crop Water Stress Using Multispectral Airborne Sensors. HortTechnology 7(1), January-March (1997)

13. Dupin, S., Gobrecht, A., Tisseyre, B.: Airborne thermography of vines canopy: effect of the atmosphere and mixed pixels on observed canopy temperature. UMR ITAP, Montpellier SupAgro, bat. 21, 2 place Viala, 34060 Montpellier, France (2011)

14. Gao, L., Cheng, M., Tang, J.: A Wireless Greenhouse Monitoring System based on Solar Energy. Telkomnika 11(9), 5448-5454 (2013) 